\title{
Intakes and sources of energy, macronutrients and dietary fibre in older Irish adults aged $\geqslant 65$ years
}

\author{
K. Evans ${ }^{1}$, L. Kehoe ${ }^{1}$, J. Walton ${ }^{1}$, B.A. McNulty ${ }^{2}$, A.P. Nugent ${ }^{2}$ and A. Flynn ${ }^{1}$ \\ ${ }^{1}$ School of Food and Nutritional Sciences, University College Cork, Cork, Republic of Ireland and ${ }^{2} U C D$ Institute of \\ Food and Health, University College Dublin, Dublin 4, Republic of Ireland
}

Adequate energy and macronutrient intakes are important for disease prevention and health maintenance in older adults. The objective of this analysis was to estimate usual intakes and sources of energy, macronutrients and dietary fibre in older Irish adults. Analysis was based on the National Adult Nutrition Survey (NANS) (2008-2010) (www.iuna.net). A 4 day semi-weighed food record was used to collect food and beverage intake data from a nationally representative sample of 1500 adults ( 226 aged $\geqslant 65$ years). Nutrient intakes were estimated using UK and Irish food composition tables. Usual intakes of nutrients were calculated via the NCI-method using SAS $^{\odot}$ Enterprise Guide ${ }^{(1)}$ and sources were calculated using SPSS $^{\mathrm{TM}}$.

Table 1. Mean daily intake of energy, macronutrients and dietary fibre in Irish adults aged $\geqslant 65$ years

\begin{tabular}{|c|c|c|c|c|c|c|c|c|c|}
\hline \multirow[b]{2}{*}{ Nutrient } & \multicolumn{3}{|c|}{ All $(n=226)$} & \multicolumn{3}{|c|}{ Men $(n=106)$} & \multicolumn{3}{|c|}{ Women $(n=120)$} \\
\hline & Mean & SD & Median & Mean & SD & Median & Mean & SD & Mediar \\
\hline Energy (Kcal) & 1778 & 490 & 1736 & 1996 & 475 & 1963 & 1585 & 417 & 1548 \\
\hline$(\mathrm{kJ})$ & 7450 & 857 & 6977 & 8335 & 220 & 8334 & 6668 & 195 & 6665 \\
\hline Protein $(\mathrm{g})$ & 77.7 & $19 \cdot 7$ & $76 \cdot 2$ & 85.9 & $19 \cdot 1$ & $84 \cdot 8$ & $70 \cdot 4$ & $17 \cdot 3$ & $69 \cdot 0$ \\
\hline (g/kg/body weight) & 0.97 & 0.55 & 0.88 & 0.94 & $0 \cdot 54$ & $0 \cdot 85$ & 0.99 & $0 \cdot 56$ & $0 \cdot 90$ \\
\hline$(\% \mathrm{TE})$ & $18 \cdot 2$ & $2 \cdot 8$ & $18 \cdot 0$ & $18 \cdot 0$ & $2 \cdot 7$ & $17 \cdot 8$ & $18 \cdot 3$ & $2 \cdot 8$ & $18 \cdot 2$ \\
\hline Carbohydrate (g) & 208 & 63 & 203 & 227 & 62 & 223 & 191 & 58 & 186 \\
\hline$(\% \mathrm{TE})$ & $44 \cdot 4$ & $5 \cdot 5$ & $44 \cdot 4$ & $43 \cdot 2$ & $5 \cdot 3$ & $43 \cdot 2$ & $45 \cdot 5$ & $5 \cdot 4$ & $45 \cdot 5$ \\
\hline Total Sugars (g) & $86 \cdot 3$ & $36 \cdot 5$ & $81 \cdot 2$ & $89 \cdot 5$ & $37 \cdot 0$ & $84 \cdot 9$ & $83 \cdot 4$ & $35 \cdot 8$ & $78 \cdot 4$ \\
\hline Total Fat (g) & 67.8 & $22 \cdot 1$ & $65 \cdot 6$ & $75 \cdot 5$ & $22 \cdot 1$ & $73 \cdot 5$ & $61 \cdot 1$ & $19 \cdot 7$ & $59 \cdot 0$ \\
\hline$(\%$ TE) & $33 \cdot 8$ & $4 \cdot 8$ & $33 \cdot 7$ & $33 \cdot 6$ & $4 \cdot 7$ & $33 \cdot 5$ & $34 \cdot 0$ & $4 \cdot 8$ & $33 \cdot 9$ \\
\hline Saturated Fat (g) & $27 \cdot 6$ & $10 \cdot 2$ & $26 \cdot 4$ & $31 \cdot 1$ & $10 \cdot 4$ & $30 \cdot 0$ & $24 \cdot 6$ & $9 \cdot 0$ & $23 \cdot 4$ \\
\hline$(\%$ TE $)$ & $13 \cdot 7$ & $2 \cdot 9$ & $13 \cdot 6$ & 13.9 & 2.9 & $13 \cdot 7$ & 13.6 & $2 \cdot 9$ & $13 \cdot 5$ \\
\hline Monounsaturated fat (g) & $24 \cdot 0$ & $8 \cdot 1$ & $23 \cdot 1$ & 26.8 & $8 \cdot 1$ & $26 \cdot 1$ & $21 \cdot 5$ & $7 \cdot 1$ & $20 \cdot 6$ \\
\hline$(\% \mathrm{TE})$ & 11.9 & 1.9 & $11 \cdot 8$ & 11.9 & 1.9 & $11 \cdot 9$ & 11.9 & 1.9 & $11 \cdot 8$ \\
\hline Polyunsaturated fat $(\mathrm{g})$ & 11.6 & $4 \cdot 3$ & $11 \cdot 0$ & $12 \cdot 3$ & $4 \cdot 4$ & $11 \cdot 8$ & 10.9 & $4 \cdot 0$ & $10 \cdot 3$ \\
\hline$(\%$ TE $)$ & $5 \cdot 8$ & $1 \cdot 5$ & $5 \cdot 7$ & $5 \cdot 5$ & 1.4 & $5 \cdot 4$ & $6 \cdot 1$ & $1 \cdot 5$ & 5.9 \\
\hline Dietary Fibre (g) & $19 \cdot 0$ & $6 \cdot 7$ & $18 \cdot 3$ & $19 \cdot 6$ & 6.7 & 18.9 & $18 \cdot 6$ & $6 \cdot 6$ & 17.9 \\
\hline
\end{tabular}

Table 2. Key sources of energy, macronutrients and dietary fibre

\begin{tabular}{ll} 
Energy & Meats $(17 \%)$, breads $(17 \%)$, milk \& yoghurt $(8 \%)$, potatoes $(7 \%)$ \\
Protein & Meats $(38 \%)$, breads $(14 \%)$, milk \& yoghurt $(11 \%)$, fish $(9 \%)$ \\
Carbohydrate & Breads $(27 \%)$, potatoes $(12 \%)$, fruit \& fruit juices $(10 \%)$, breakfast cereals $(9 \%)$ \\
Total Fat & Meats $(24 \%)$, fat spreads \& oils $(17 \%)$, milk \& yoghurt $(9 \%)$, biscuits, cakes \& pastries $(7 \%)$ \\
Saturated Fat & Meats $(23 \%)$, fat spreads \& oils $(17 \%)$, milk \& yoghurt $(13 \%)$, biscuits, cakes \& pastries $(8 \%)$ \\
Dietary Fibre & Breads $(29 \%)$, vegetables $(18 \%)$, fruit \& fruit juices $(15 \%)$, potatoes $(12 \%)$ \\
\hline
\end{tabular}

Mean intakes of protein were above the Population Reference Intake (PRI) of $0.83 \mathrm{~g} / \mathrm{kg}$ body weight per day proposed by the European Food Safety Authority (EFSA $)^{(2)}$. Mean daily intakes of carbohydrate and total fat were approaching or within the reference intake ranges $(45-60 \% \mathrm{TE}, 20-35 \% \mathrm{TE})^{(3,4)}$; however, the mean intake of saturated fat was higher than recommended $(\leqslant 10 \%$ $\mathrm{TE})^{(5)}$. Furthermore mean daily intake of dietary fibre was lower than the adequate intake of $25 \mathrm{~g} / \mathrm{d}$ proposed by EFSA ${ }^{(3)}$. These findings may be useful for developing dietary strategies to improve macronutrient and dietary fibre intake in older Irish adults.

This research was funded by the Department of Agriculture, Food and the Marine under the Nutridata Project 13 F $542 ; 2014$ 2016.

1. Tooze JA, Kipnis V, Buckman DW et al. (2010) Stat Med. 29 (27): 2857-68.

2. EFSA (2012) EFSA $J 10$ (2): 2557

3. EFSA (2010) EFSA $J \mathbf{8}$ (3): 1462.

4. EFSA (2010) EFSA $J \mathbf{8}$ (3): 1461

5. UK Department of Health (1991) Dietary Reference Values for Food Energy and Nutrients for the United Kingdom. London: HMSO. 